\title{
Disorder, clustering, and localization effects in amorphous carbon
}

\author{
J. D. Carey* and S. R. P. Silva \\ Nanoelectronics Centre, Advanced Technology Institute, School of Electronics and Physical Sciences, University of Surrey, \\ Guildford, GU2 7XH, United Kingdom
}

(Received 2 August 2004; revised manuscript received 28 September 2004; published 16 December 2004)

\begin{abstract}
The nanostructure of amorphous carbon thin films is described in terms of a disordered nanometer-sized conductive $s p^{2}$ phase embedded in an electrically insulating $s p^{3}$ matrix. It is shown that the degree of clustering and disorder within the $s p^{2}$ phase plays a determining role in the electronic properties of these films. Clustering of the $s p^{2}$ phase is shown to be important in explaining several experimental results including the reduction of the electron spin resonance linewidth with increasing spin density and the dispersion associated with the width of the Raman active $G$ band. The influence of structural disorder, associated with $s p^{2}$ clusters of similar size, and topological disorder, due to undistorted clusters of different sizes, on both spin density and Raman measurements, is discussed. An extension of this description to intercluster interactions to explain some of the electrical transport and electron field emission behavior is also presented.
\end{abstract}

DOI: 10.1103/PhysRevB.70.235417

PACS number(s): 71.23.Cq, 79.70.+q, 85.45.Db, 73.61.Jc

\section{INTRODUCTION}

Considerable research effort into amorphous group IV semiconductors has been spurred on by the ability to control their electronic properties by varying the deposition conditions and a capability to deposit films over large areas. Amorphous carbon $(a-\mathrm{C})$ thin films have the added advantage that they can be deposited at low substrate temperatures where it is possible to achieve hard films with rms roughnesses of less than $1 \mathrm{~nm}$ over large areas. ${ }^{1,2}$ This has led to applications such as hard disk and biomedical coatings. Less success has been forthcoming in the development of active electronic applications of $a-\mathrm{C}$ and hydrogenated $a-\mathrm{C}(a-\mathrm{C}: \mathrm{H})$ films ${ }^{3}$ and as a consequence it is necessary to examine the electronic properties and the effects of disorder and localization at a nanometer level. Unlike other group IV tetrahedral amorphous semiconductors, such as amorphous silicon or germanium, the different hybridizations of $\mathrm{C}$ atoms allows for the formation of $s p^{2}$ as well as $s p^{3}$ bonding. The $s p^{2}$ hybridized carbon atoms tend to exist as nm-sized clusters in the form of graphitic rings or olephinic chains giving rise to $\pi$ and $\pi^{*}$ bands, whereas the $s p^{3}$ component tends to be in the form of aliphatic chains. The optoelectronic properties of these films are usually discussed in terms of transitions between occupied $\pi$ states to unoccupied $\pi^{*}$ states with the $\sigma$ and $\sigma^{*}$ states (associated with $s p^{3}$ C) further separated from each other. It has been shown ${ }^{4}$ that it is possible to represent the occupied $\pi$ and unoccupied $\pi^{*}$ bands by Gaussian functions centered at $E_{\pi\left(\pi^{*}\right)}$. The usual methods for extracting parameters such as the (parabolic) Tauc gap or the (exponential) Urbach energy are used, although there is a growing realization that this approach may be too simplistic for this composite amorphous system. ${ }^{5}$

In most disordered semiconductors, the Tauc band gap corresponds to optical transitions between extended states, and in the case of $a-\mathrm{C}: \mathrm{H}$, in the absence of disorder, the Tauc gap is given by the separation between the $\pi$ and $\pi^{*}$ bands and gives a magnitude of $2 E_{\pi}$. The larger the average size of the $s p^{2}$ cluster, the smaller the Tauc gap. An alterna- tive band gap that is sometimes employed is to measure the energy at which the absorption coefficient is $10^{4} \mathrm{~cm}^{-1}$; this is known as the $E_{04}$ gap. Values associated with the $E_{04}$ gap tend to be larger than the Tauc gap as the absorption associated with $10^{4} \mathrm{~cm}^{-1}$ tends to occur in states beyond the $\pi$ and $\pi^{*}$ band edges. Transitions between extended-to-localized states in amorphous semiconductors are usually characterized by the Urbach energy, $E_{U}$, which is determined by fitting an exponential function to the slope of the absorption edge. In the case of low defect density $a-\mathrm{Si}: \mathrm{H}$ the valence band tail has an exponential slope of about $45 \mathrm{meV}$, which is larger than the slope of the conduction band $(25 \mathrm{meV})$. In this manner the Urbach energy of $55 \mathrm{meV}$ for low defect density $a-\mathrm{Si}: \mathrm{H}$ is mainly determined by the valence band and associated with low-lying defect states. In this way the Urbach energy is a measure of disorder.

A second measure of disorder, based upon bond angle distortion, is the width (full width half maximum) of the Raman active $G$ band, $\Gamma_{G}$. This Raman signal is found at around $1580 \mathrm{~cm}^{-1}$ and originates from the $E_{2 g}$ vibration between $s p^{2} \mathrm{C}$ bonds. ${ }^{6}$ Several complementary optical ${ }^{7-9}$ and Raman studies, ${ }^{6,10}$ have revealed that as the Tauc gap increases the Urbach energy increases monotonically but that the width of the $G$ band undergoes a maximum about $1.5 \mathrm{eV}$, as shown in Fig. 1(a). In this respect if the Urbach energy and the width of $G$ band are valid measures of disorder in the $a-\mathrm{C}: \mathrm{H}$ system, then they measure different aspects or types of disorder. Robertson has concluded that the Urbach energy is a measure of an inhomogeneous disorder associated with different $s p^{2}$ cluster sizes, whereas the $G$ band width has been attributed to a homogeneous disorder associated with bond angle disorder. ${ }^{2}$ However, this interpretation is unable to explain

(i) why the largest values of $E_{U}$ are observed at the highest Tauc gaps [Fig. 1(a)] where the cluster sizes are smallest and as a result there is only a small limited number of different cluster conformations possible,

(ii) the observation of a maximum in the $G$ band linewidth with the Tauc gap [Fig. 1(b)], 


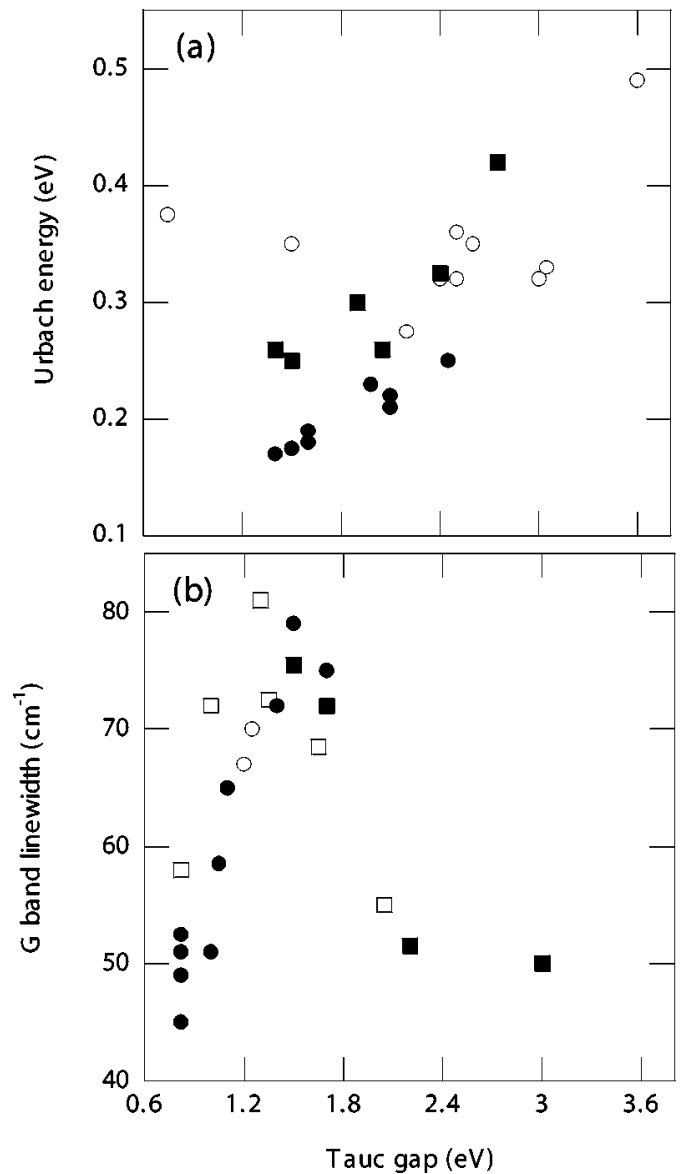

FIG. 1. Variation of (a) Urbach energy from (ם) from Ref. 7, (O) from Ref. 8, and (O) from Ref. 9 and (b) linewidth of the Raman $G$ band for films deposited from $\mathrm{CH}_{4}(-), \mathrm{C}_{6} \mathrm{H}_{6}(\square)$, and $\mathrm{C}_{4} \mathrm{H}_{10}(\square)$ from Ref. 10 and from $\mathrm{CH}_{4}(\bigcirc)$ from Ref. 6 as a function of the optical Tauc gap.

(iii) why the $G$ band position is also dependant on the excitation wavelength, with the $G$ band position tending to saturate at $\sim 1600 \mathrm{~cm}^{-1}$ for excitation wavelengths below $350 \mathrm{~nm}$ and the peak shifting to lower energies at longer wavelengths, as shown in Fig. 2(a), and

(iv) the observation that the $G$ band linewidth depends on the excitation wavelength with the narrower values of $\Gamma_{G}$ observed at increasing excitation energy as seen in Fig. 2(b).

Since the Raman spectra are a result of resonant excitation of $s p^{2}$ clusters, dispersion of the $G$ peak reflects excitation of clusters with different band gaps. The saturation of the $G$ band position at $\sim 1600 \mathrm{~cm}^{-1}$ in Fig. 2(a) reflects the maximum possible Raman shift for $\mathrm{C}$ atoms bonded in $s p^{2}$ rings (noting that higher Raman shifts are possible for $s p^{2} \mathrm{C}$ atoms found in chains, with shorter bond lengths). ${ }^{6}$ The observation of dispersion of the Raman linewidth implies that there is an inhomogeneous distribution in bond angle disorder. Furthermore, the reduction of the $G$ width with increasing excitation energy implies that there is a narrower distribution of higher gap $s p^{2}$ states, in contradiction with the suggestion that there must be a larger distribution of $s p^{2}$ states required for the large Urbach energy.

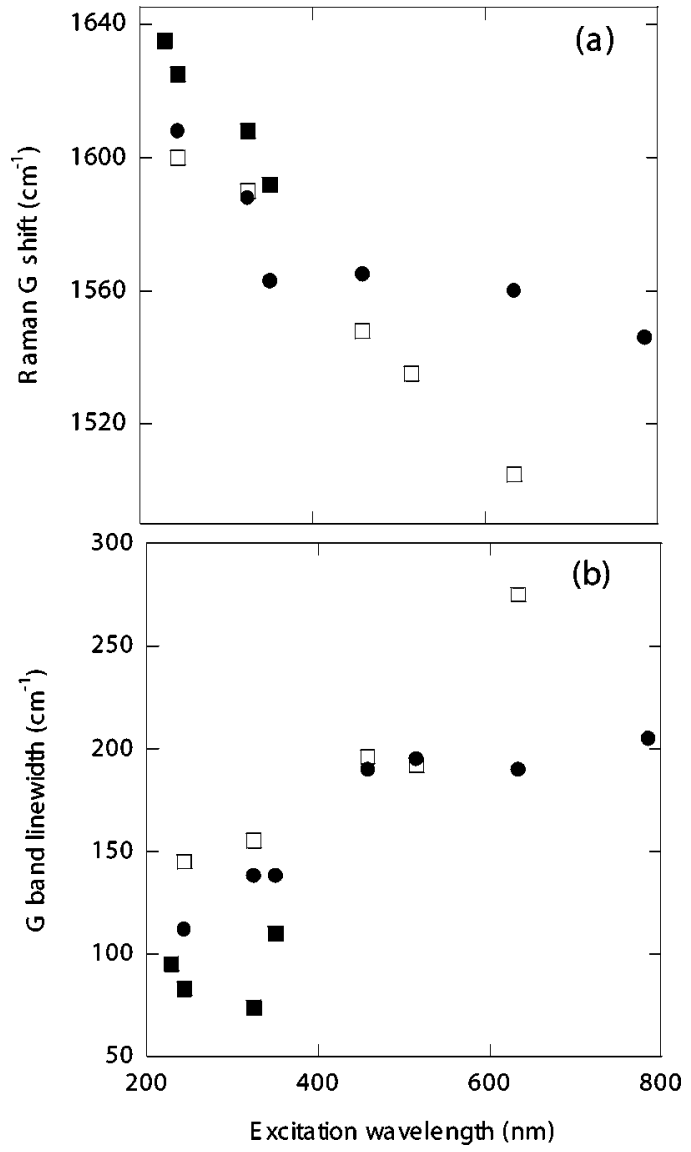

FIG. 2. Variation of (a) the $G$ band Raman shift and (b) $G$ band linewidth for a series of polymerlike $a-\mathrm{C}: \mathrm{H}$ films $(\boldsymbol{\square})$, diamondlike $a-\mathrm{C}: \mathrm{H}(\square)$, and graphitic carbon $(\bullet)$ thin films as a function of Raman excitation wavelength (Ref. 6).

In order to understand the types of disorder that are present it is also worthwhile highlighting the role of electron spin resonance (ESR) active "defects." A high concentration of paramagnetic defects $\left(10^{20} \mathrm{~cm}^{-3}\right)$ tends to be found in films deposited under energetic conditions, such a high negative self-bias found in plasma enhanced chemical vapor deposition (PECVD). ${ }^{11}$ Films grown with a high $\mathrm{H}$ content and low internal stresses tend to have much lower defect densities, typically $\sim 10^{17} \mathrm{~cm}^{-3} .{ }^{12}$ The term "defect" is perhaps a misnomer since the ESR active species are generally agreed to result from different configurations of $s p^{2}$ clusters. In this way a proper interpretation of the ESR data can be linked to optical band gap, Raman data as well as from nuclear magnetic resonance measurements. The exploration of these intracluster interactions and how they are related to disorder is one of the aims of this paper. The paper is organized into three sections. First, the importance of disorder and localization of the electron wave function in the $a-\mathrm{C}$ system is introduced. Second, the structure and bonding on a nanoscale is explored in order to understand the intracluster interactions and evidence of clustering found in $a-\mathrm{C}: \mathrm{H}$ and $a-\mathrm{C}$ films. Finally, we show how the intercluster interactions can be altered and their resultant effects on the electronic properties of the films. 


\section{DISORDER AND LOCALIZATION EFFECTS IN AMORPHOUS CARBON}

It is now well established that in the case of metals the conductivity is determined by diffusive electronic states. In the case of amorphous materials, disorder plays an important role with a net reduction of the mobility of the carriers via scattering. Anderson showed that in a sufficiently disordered one-band model no movement of carriers can take place without thermal activation. ${ }^{13}$ Tunneling between localized states becomes possible at higher temperatures and the extent of electron hopping is controlled by the properties of the midgap and tail states. Using the notation developed by Anderson, it was shown that localization can be described in terms of a tight-binding model with a single band of width $B$, which is equal to $2 z V$, where $z$ is the coordination number and $V$ is the potential well of the atom. When the magnitude of the disorder potential, $\delta V$, is larger than $B$, it is found that

$$
\delta V / B=\delta V / 2 z V>1 \text { and } \delta V / V>2 z .
$$

In the case of $a-\mathrm{C}: \mathrm{H}$, where both $\pi$ and $\sigma$ bonding is present, the calculations of Chen and Robertson showed that the entire $\pi$ band is localized with a high inverse participation ratio of the $\pi$ states that control the conduction properties. ${ }^{14}$ Unlike the case of spherical hybridized wave functions, for which a random arrangement of spheres retains a high degree of spherical wave function overlap, the planes involving $\pi$ bonds with $p$ hybridized states in $a$-C end up being close to orthogonal to each other. This results in little interaction as the potential well overlap of each atom tends to zero. In terms of Eq. (1) this means that the potential $V$ is now comparable to the disorder term $\delta V$, resulting in electron localization. One consequence of a localized wave function is that the photoluminescence (PL) emission is predicted to be highly polarized, a prediction that was subsequently confirmed by polarized PL measurements. ${ }^{15}$ Another experimental result to note is the localization length measured ${ }^{16}$ in low-defect-containing $a-\mathrm{C}: \mathrm{H}$ films, which was about $1.5 \mathrm{~nm}$. This value can be compared with that found in $a-\mathrm{Si}$, which is typically around $10-12 \mathrm{~nm}$. In the case of $a-\mathrm{Si}$, in which only $\sigma$ bonds are present, localization only occurs at the tail states of both conduction and valence bands.

The increase in the Urbach energy with increasing $\pi$ - $\pi^{*}$ separation and small numbers of possible $s p^{2}$ cluster configurations coupled with the dispersion in the $G$ linewidth have led Fanchini and Tagliaferro to conclude that the Urbach energy in amorphous carbon is not a good measure of disorder. ${ }^{17}$ Furthermore, they went on to propose two types of disorder:

(i) A structural disorder associated with clusters of the same size but with different amounts of distortion. This type of inhomogeneous disorder would increase the Urbach energy as well as broaden the $G$ band linewidth.

(ii) Topological disorder arising from undistorted clusters but of different sizes. This would also broaden the Urbach energy but have no effect on the $G$ linewidth.

By modeling the $\pi-\pi^{*}$ bands as Gaussian bands, Fanchini and Tagliaferro showed that it is the ratio of the Gaussian width to the $\pi$ band energies that is important, not simply the width of the $\pi$ bands. This gives rise to a disorder-induced narrowing of the Tauc optical gap. As a consequence the difference between distorted and undistorted $s p^{2}$ clusters needs to be explored. The location of the $s p^{2}$ clusters in the energy gap depends on two factors: whether they consist of even or odd numbers of carbon atoms and whether they are distorted. Undistorted even numbered clusters will give rise to states near the Fermi level $\left(E_{\mathrm{F}}\right)$ only if they are sufficiently large. This differs from the case of odd numbered clusters, which can give rise to gap states even if composed of a small number of atoms. In general, distorted clusters give rise to states that are closer to $E_{\mathrm{F}}$ than undistorted clusters. In this manner ESR can give a measure of the density of $s p^{2}$ states at the Fermi level, provided that a majority of these states have a net unpaired electron spin present. Note that measurements of ESR spin densities do not themselves measure the $s p^{2}$ content. For example, electron energy loss spectroscopy measurements made on PECVD grown films showed that the $\pi$ bonds per carbon atom, taken to be the $s p^{2}$ content, varied from $63 \%$ to $72 \%$ for a change of negative self-bias from 100 to $265 \mathrm{~V}$. This was accompanied by an increase in the spin density from $4.9 \times 10^{19}$ to $1.5 \times 10^{20} \mathrm{~cm}^{-3}$. However, whilst increasing the negative self bias to $540 \mathrm{~V}$ the spin density hardly changed $\left[(2-3) \times 10^{20} \mathrm{~cm}^{-3}\right]$, the $s p^{2}$ content increased to nearly $100 \% .^{11}$

\section{CLUSTERING, DISORDER, AND INTRACLUSTER EFFECTS IN AMORPHOUS CARBON}

In order to examine the degree of clustering present in $a$ $-\mathrm{C}: \mathrm{H}$ films, solid state nuclear magnetic resonance (NMR) has been employed. This has the distinct advantage over Raman spectroscopy in that NMR is able to quantify the relative amounts of $s p^{2}$ and $s p^{3} \mathrm{C}$ bonding along with the degree of protonation of each type of atom. The biggest disadvantage of NMR is the relatively large amount of sample required since only the ${ }^{13} \mathrm{C}$ nucleus, with an abundance of $1.1 \%$, possesses a nonzero nuclear spin of $\frac{1}{2}$. Jäger et al. used cross-polarization ${ }^{13} \mathrm{C}$ NMR to measure the effects on the proton relaxation times in different environments of films deposited from acetylene and benzene and found evidence ${ }^{18}$ for a two-component ${ }^{1} \mathrm{H}$ spin-lattice relaxation with time constants of 14 and $120 \mathrm{~ms}$. This was interpreted as being due to the presence of two differently relaxing proton systems in which the shorter relaxation time originates from $\mathrm{CH}$ groups in the $s p^{2}$ and $s p^{3}$ matrix. The long $T_{1}$ time corresponds to short $\mathrm{CH}_{2}$ polymer units with a $s p^{3}$ configuration. In this description the two environments are separated by regions of nonhydrogenated $s p^{2}$ C. Further interpretation about the nature of the (hydrogenated) $s p^{2}$ component of the films comes from the observation of a virtually temperatureindependent proton relaxation time of $\sim 10 \mathrm{~ms}$ for films deposited by rf glow discharge from benzene at a self-bias voltage of $-200 \mathrm{~V}$. The temperature independence of the shorter $T_{1}$ component is consistent with the nuclei being relaxed via spin diffusion to paramagnetic centers. It is therefore the degree of electron aggregation or clustering associated with paramagnetic centers that determines the proton 
relaxation. The influence of spin diffusion to paramagnetic centers as an explanation of the temperature independence of the spin-lattice relaxation times implies that an understanding of the facts that affect the spin density and linewidth are important.

Films of $a-\mathrm{C}: \mathrm{H}$ were deposited on either the driven or earthed electrode of a Plasma Technology DP800 capacitively coupled radio-frequency $13.56 \mathrm{MHz}$ plasma-enhanced chemical vapor deposition system. Films were grown on Corning 7059 glass for optical measurements and highresistivity substrates for ESR measurements. During deposition, feed gases of methane [30 $\mathrm{sccm}$ (standard cubic centimeters per minute)] with $\mathrm{He}(75 \mathrm{sccm})$ were used and the total deposition pressure was 200 mTorr. Film thicknesses were measured using ellipsometry. Films grown at low negative self-biases are soft, low stress, low density $\left(\sim 1.5 \mathrm{~g} \mathrm{~cm}^{-3}\right)$, possess high $\mathrm{H}$ contents (up to 40 at. \%) and are optically transparent. These films are referred to as polymerlike amorphous carbon (PAC) films. Diamondlike carbon (DLC) films are hard, have a high Young's modulus, high density, possess lower hydrogen contents, have relatively large dielectric constants, have high stress (overconstrained networks), and lower optical band gaps. In the limit of films exhibiting high $s p^{2}$ content, graphitic carbon (GC) is formed with close to zero optical gap. ${ }^{1}$ Figure 3 shows the variation of the spin density, $N_{\mathrm{s}}$, and peak-to-peak linewidth, $\Delta B_{\mathrm{pp}}$, with negative self-bias for a series of films deposited from $\mathrm{CH}_{4}$ as a hydrocarbon source gas. Two other sets reported in the literature are also presented. ${ }^{7,11}$ It is evident that the spin density rises from a low value of $\sim 10^{17} \mathrm{~cm}^{-3}$ and saturates at $10^{20} \mathrm{~cm}^{-3}$. The ESR linewidth initially increases, often as the spin density increases, but at higher biases the linewidth decreases before saturating.

Since the spin densities under high bias conditions reported are typically $\sim 10^{20} \mathrm{~cm}^{-3}$, corresponding to $\sim 1$ at. $\%$ of the material present, we may reasonably assume that the ESR signal can be considered as originating from a dilute paramagnetic material. It is therefore possible to apply the theory of Abragam to calculate the contribution to $\Delta B_{\mathrm{pp}}$ from the dipole-dipole interaction between like spins. ${ }^{19}$ Such a broadening mechanism will produce a Lorentzian ESR line shape, a prediction that was subsequently observed. ${ }^{11}$ At lower biases, the line shape is often Gaussian, characteristic of inhomogeneous broadening, and usually attributed to unresolved $\mathrm{H}$ hyperfine interactions. The presence of this extra contribution to the linewidth complicates the discussion of the linewidth at low values of $N_{\mathrm{s}}$. Assuming a spin concentration $N_{\mathrm{s}}$, measured in $\mathrm{cm}^{-3}$, the contribution to the dipolar interaction $\Delta B_{\mathrm{pp}}$, measured in $\mathrm{mT}$, can be given as ${ }^{11}$

$$
\Delta B_{\mathrm{pp}}=\frac{4 \pi^{2}}{9} g \mu_{B} N_{\mathrm{s}}
$$

Barklie et al. applied Eq. (2) for a typical C-related $g$ value of 2.0025 to obtain the expression $\Delta B_{\mathrm{pp}}=8.12 \times 10^{-21} N_{\mathrm{s}}$. Figure 4 shows the variation of $\Delta B_{\mathrm{pp}}$ against $N_{\mathrm{s}}$ directly for the same three data sets reported in Fig. 3, as well the predicted linewidth (dashed line) based solely on dipolar broadening using Eq. (2). It is apparent that significant reductions

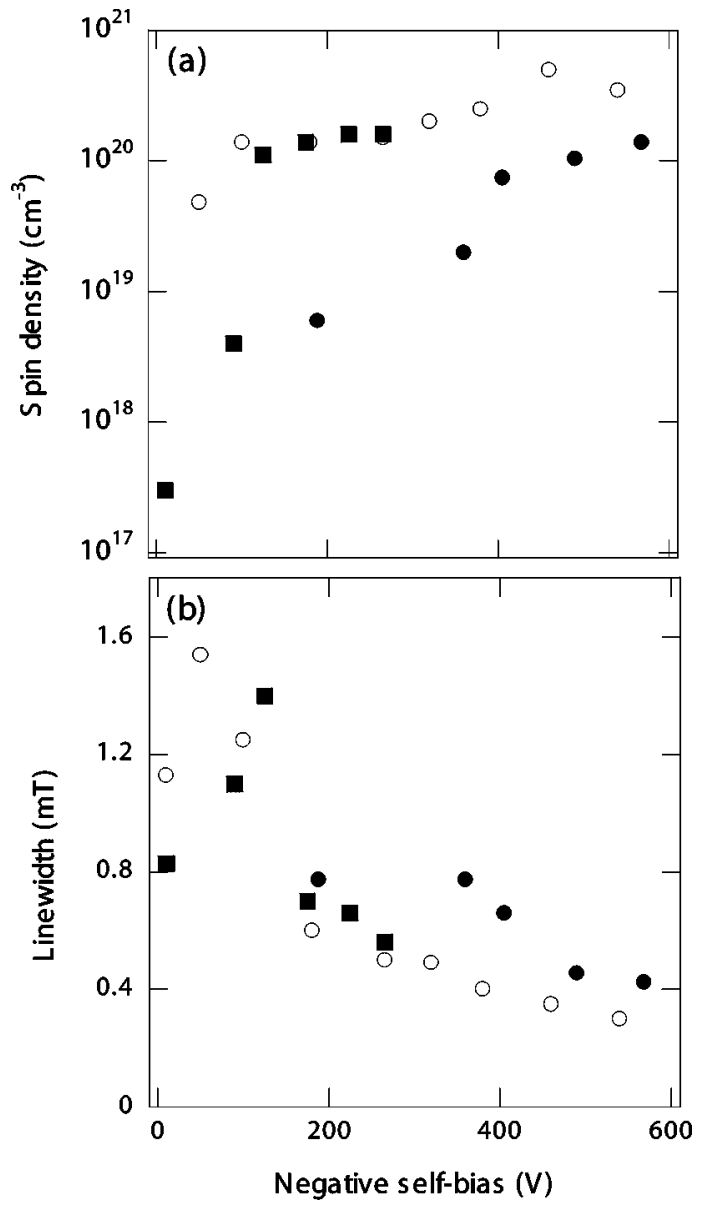

FIG. 3. Variation of (a) spin density and (b) peak-to-peak linewidth with negative self-bias for a series of DLC films (ם) and from Ref. $7(\mathbf{O})$ and Ref. $11(\bigcirc)$.

in $\Delta B_{\mathrm{pp}}$ are observed at high $N_{\mathrm{s}}$, indicating an additional mechanism is responsible for reductions in the linewidth. This additional interaction is due to the motional averaging of the electron wave function within the $s p^{2}$ cluster. As the $s p^{2}$ cluster increases in size the probability of the electron being associated with a particular atom decreases. This spread out or delocalization of the wave function as the cluster size increases is accompanied by a general reduction in the Tauc gap. Evidence of delocalization can also be found in an examination of the spin resonance relaxation times. The spin-lattice relation time, $T_{1}$, decreases from $3 \times 10^{-5} \mathrm{~s}$ for PAC films down to $2 \times 10^{-7} \mathrm{~s}$ for DLC films, which implies a greater interaction between the spin system and lattice. ${ }^{11}$ At the same time the spin-spin relaxation times increase, reflecting a change in the exchange frequency. It has been reported ${ }^{11}$ that in films with a high $s p^{2}$ content that the exchange frequency is approximately $\sim 10^{10} \mathrm{rad} \mathrm{s}^{-1}$.

Further evidence confirming the influence of clustering can be found from NMR measurements. Tamor et al. estimated the protonated and nonprotonated $\mathrm{C}$ concentration with either $s p^{2}$ or $s p^{3}$ hybridization. ${ }^{20}$ At a self-bias of $-500 \mathrm{~V}$, the $\mathrm{H}$ content was estimated to be 36 at. \% and the fraction of protonated $s p^{3} \mathrm{C}$ atoms to be 0.16 , nonprotonated $s p^{3} \mathrm{C}$ atoms to be 0.20 , protonated $s p^{2} \mathrm{C}$ atoms to be 0.25 , and nonprotonated $s p^{2} \mathrm{C}$ atoms to be 0.37 . For a film density 


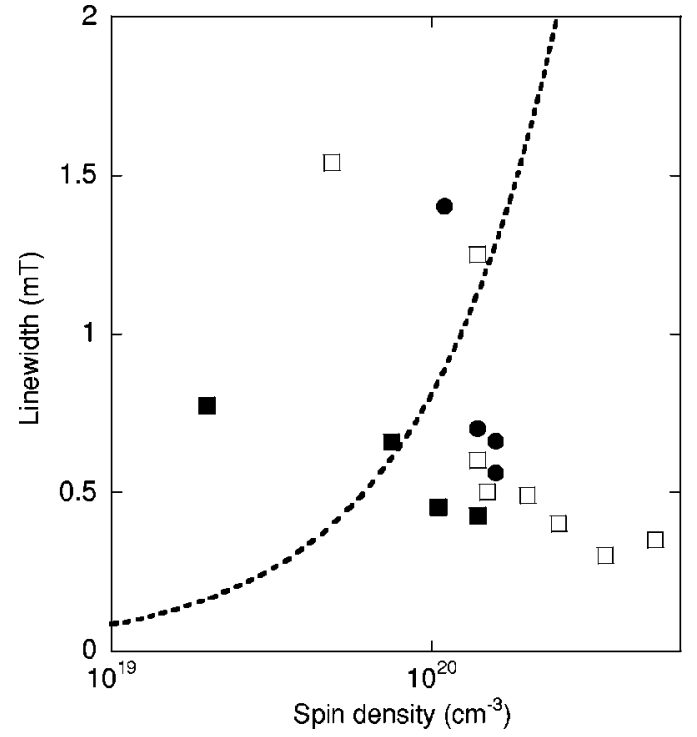

FIG. 4. Variation of peak-to-peak linewidth with spin density for a series of DLC films ( $)$ and from Ref. 7 (@) and Ref. $11(\bigcirc)$. The dashed line represents the predicted dipolar contribution to the linewidth based on Eq. ((2)).

of $1.7 \mathrm{~g} \mathrm{~cm}^{-3}$ this result suggests there are $5.1 \times 10^{22} s p^{2} \mathrm{C}$ atoms $\mathrm{cm}^{-3}$ in the film. If the signal observed from ESR is associated with $s p^{2} \mathrm{C}$ centers, then for a value of $N_{\mathrm{s}}$ of $10^{20} \mathrm{~cm}^{-3}$, this corresponds to 1 spin per $510 s p^{2} \mathrm{C}$ atoms or equivalent to 1 spin per 300 atoms of nonprotonated $s p^{2} \mathrm{C}$ atoms. If these spins were randomly distributed throughout the film cooperative effects, such as exchange, should not occur. Since exchange effects have been attributed to films grown at high bias, the combined use of NMR and ESR confirms and quantifies the clustering of the $s p^{2}$ phase that has taken place.

In addition to the concentration of the $s p^{2}$ clusters, the size of the $s p^{2}$ clusters is therefore an important feature of this description. Indeed there have been attempts to infer the cluster size and shape using visible Raman spectroscopy. ${ }^{21}$ As a result considerable interest was generated by the recent observation $^{22}$ of apparent anisotropy in the ESR signal of defects in DLC films measured at high frequency $(94 \mathrm{GHz})$ and low temperature $(4 \mathrm{~K})$. A fully amorphous material with a single spin- $\frac{1}{2}$ paramagnetic center should exhibit a single isotropic line. The presence of strong exchange results in a single line whose linewidth is exchange narrowed below that predicted by Eq. (2). In Ref. 22 it was proposed that the ESR signal could be attributed to the sum of an anisotropic powder spectrum with axial $g$ values of $g_{\|} \sim 2.005$ and $g_{\perp}$ $\sim 2.0025$ and one symmetric unresolved line with $g$ $=2.0025$. From ESR studies of nanocrystalline graphite it is known that the $g$ tensor is anisotropic and the $g$ values depend on the average crystallite size, such that the observed value of $g_{\|}$of 2.005 being associated ${ }^{23}$ with a crystallite size of less than $7.5 \mathrm{~nm}$. In this way it was believed that ESR was providing a way to quantify the size of the nanoclusters. This is an extremely attractive feature since it has been proposed that the ratio of the intensities of the $G$ and $D$ Raman bands (located at $1350 \mathrm{~cm}^{-1}$ and associated with the $A_{1 g}$ breathing

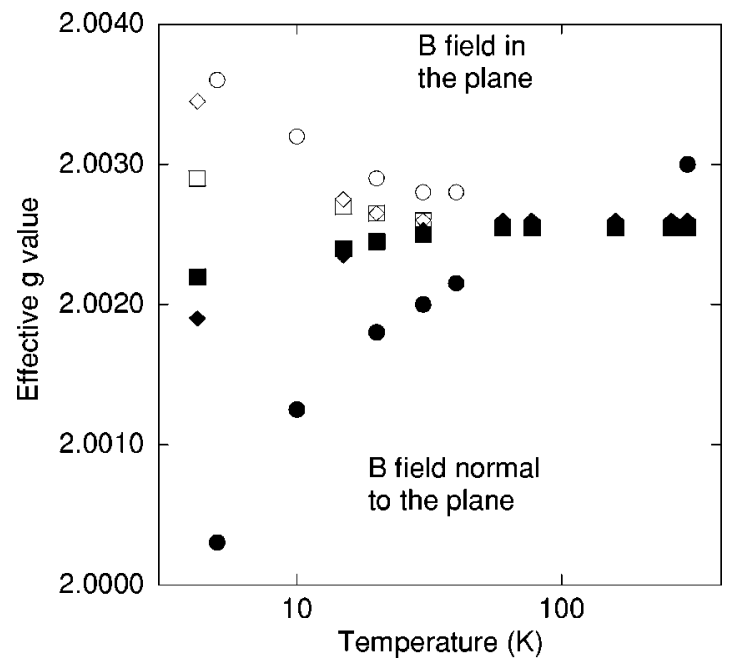

FIG. 5. Variation of the effective $g$ value for the magnetic field in the plane of (open symbols) and perpendicular to (closed symbols) the film with measurement temperature for a series of DLC films after growth (circles) (Ref. 22 ), after implantation with $\mathrm{Ni}$ (squares) and W (diamonds) (Ref. 26).

mode of six-fold $s p^{2}$ rings) can be related to the in-plane correlation of disordered graphite. ${ }^{6,21}$ The reason that no anisotropy was observed at the more conventional $9 \mathrm{GHz}$ room-temperature measurement was due to the strong exchange narrowing. Unfortunately, in this analysis the role of demagnetizing fields, which at room temperature and "low" magnetic fields $(\sim 0.3 \mathrm{~T}$ for a microwave frequency of $9 \mathrm{GHz}$, corresponding to a $g=2$ center) are negligible, but must be included when operating at $4 \mathrm{~K}$ and especially at higher resonance fields required at the higher microwave frequencies. ${ }^{24,25}$ When demagnetizing fields are taken into account, an apparent anisotropy of the resonance can occur since the demagnetizing field results in an effective shift downwards of the effective $g$ value when the applied Zeeman magnetic field is perpendicular to the film. Similarly, an upshift in the $g$ value occurs when the applied field is parallel to the plane, as can be seen in Fig. 5 for a range of samples both deposited and ion implanted. ${ }^{26}$ Typically, for an applied field of $0.3 \mathrm{~T}$ and spin concentration of $10^{20} \mathrm{~cm}^{-3}$ the demagnetizing field is about $1 \mathrm{mT}$. As a result exchange interactions are still prevalent at high microwave fields.

Finally, apart from changes in deposition conditions, e.g., self bias or the addition of $\mathrm{N}$, nanoclustering within $a-\mathrm{C}$ films can be adjusted via thermal annealing. Siegal et al. annealed nonhydrogenated $a-\mathrm{C}$ films produced by pulsed laser ablation and reported the presence, via high-resolution transmission electron microscopy, of regions $3-5 \mathrm{~nm}$ in size that were $5-10 \%$ more dense that the surrounding regions. ${ }^{27}$ The as-deposited films have stresses of 5-7 GPa and a film density of $3.0 \mathrm{~g} \mathrm{~cm}^{-3}$ but near-complete stress relief had occurred and the density had dropped to $2.8 \mathrm{~g} \mathrm{~cm}^{-3}$ with thermal annealing to $600{ }^{\circ} \mathrm{C}$. Over the same annealing temperature range the optical transparency of the film decreased, demonstrating that clustering of the $s p^{2}$ within the film is increasing. By considering different bonding topologies on the basis of Raman spectroscopy, Siegal et al. concluded that 
the as-grown films consist of the small and isolated sixfold clusters but that above annealing temperatures of $300^{\circ} \mathrm{C}$ the fraction of five-membered rings begins to increase. The presence of five-membered rings was attributed to the newly formed $\pi$ bonded atoms, rather than a reduction in the population of the six-membered rings themselves. Despite the reduction in the film's density, nanoindentation measurements showed that the nanocomposite films were $15 \%$ harder than the as deposited films. Computational studies showed that within this nanocomposite film, the residual stress consists of an inhomogeneous stress distribution where the threefoldcoordinated atoms are under tensile stress but that the fourfold atoms are under a smaller compressive stress. ${ }^{28}$ During annealing small regions of high stress relax, leading to the preferential generation of threefold-coordinated atoms. The stress relief is, as a result of fourfold $s p^{3} \mathrm{C}$ bonds, being replaced by threefold $s p^{2} \mathrm{C}$ bonds. This change to the shorter $s p^{2} \mathrm{C}$ bond is irreversible under normal conditions. If the near-zero stress is achieved due to high-temperature annealing, care must the exercised to prevent an excessive thermal stress being introduced into the film on cooling back to room temperature. The thermal stress can be eliminated by growing the film at above room temperature, which introduces some tensile stress. Further evidence of the formation of clustering in tetrahedral amorphous carbon $(t a-C)$ films was reported in the fluctuation microscopy studies recently undertaken by Chen et al. ${ }^{29}$ Annealing up to $600{ }^{\circ} \mathrm{C}$ resulted in the increase of locally ordered regions, but the formation of graphitic ordering did not occur until annealing to $1000{ }^{\circ} \mathrm{C}$. Much of the research for stress relief in hard $a$-C films, especially $t a-\mathrm{C}$ films is driven by the development of carbonbased microelectromechanical systems.

\section{INTERCLUSTER INTERACTIONS IN AMORPHOUS CARBON}

The increase in intracluster delocalization also manifests itself in changes that can be associated with intercluster interactions. Specifically, we shall discuss how influence on (low-field) conductivity and (high-) field-induced electron emission into vacuum. Various transport studies of $a$-C films reveal a commonality in behavior with high resistivity associated with samples with large Tauc gap. The resistivity decreases as the Tauc gap decreases and the conductivity associated with various mechanisms such as variable range hopping in the more conductive DLC films. ${ }^{1,2}$ In the case of resistive "polymeric" amorphous carbon the conductivity has been attributed to a space charge induced current mechanism. ${ }^{1}$ Improvements in the conductivity have also been reported with nitrogen incorporation and attributed to a weak "doping" effect ${ }^{30}$ or to a reduction of the band gap through graphitization. ${ }^{31}$ In terms of postdeposition processing, transport studies of annealed films also reveal an improvement in the conductivity; however, this is almost always accompanied by significant modification to the structure of the film at a microscopic level. In this respect thermal annealing in a furnace results in an improvement in electrical properties but accompanied by global changes to the film. Ion implantation as a method of injection of thermal

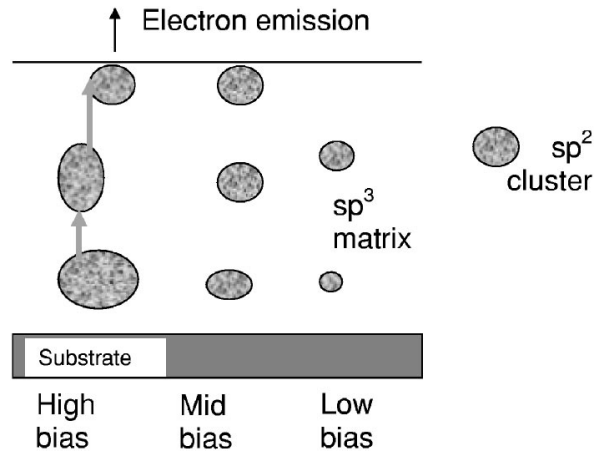

FIG. 6. Schematic of how the different bias growth conditions can give rise to a change in the size and concentration of $s p^{2}$ clusters.

energy is therefore an alternative process to modify the film properties in a highly controlled manner with precise depth control by the correct choice of ion species, ion energy, and dose. We have previously shown ${ }^{30}$ that by using high-dose $\mathrm{B}^{+}$implantation into polymerlike films, it is possible to reduce the low-field resistivity from $2 \times 10^{14} \Omega \mathrm{cm}$ (unimplanted) to $6 \times 10^{12} \Omega \mathrm{cm}\left(2 \times 10^{14} \mathrm{~B}^{+} \mathrm{cm}^{-2}\right)$. Over the same range the Tauc gap remained constant at about $2.6 \mathrm{eV}$, indicating that no significant increase in the mean $s p^{2}$ cluster size has occurred. For doses greater than $2 \times 10^{14} \mathrm{~cm}^{-2}$, a fall in the optical gap finally ends with a total collapse to $0.2 \mathrm{eV}$ at a high dose $\left(2 \times 10^{16} \mathrm{~B}^{+} \mathrm{cm}^{-2}\right)$ and the resistivity concomitantly decreases to $5 \times 10^{6} \Omega \mathrm{cm}$. The improvement in the electrical characterization has been attributed to improvements in the cluster-cluster interaction via a hopping related mechanism as shown in Fig. 6.

During low-dose implantation, localized heating results in localized $s p^{2}$ clusters, which remain small (as evidenced by the absence of a reduction in the Tauc gap) - as shown in Fig. 6. Before the formation of damage cascades, the concentration of the induced sites will be proportional to the ion dose. This nanoclustering of the $s p^{2}$ sites, which maintains the wide gap, increases the conductivity by decreasing the hopping distance. At higher doses, nanostructuring of the bulk of the film occurs and the band gap decreases. As a result, ion implantation demonstrates that it is possible to improve the conductivity without inducing extensive graphitation of the film. Further evidence for the idea of dielectric inhomogeneity of conductive $s p^{2}$ clusters embedded in a more insulating matrix can be found in the high-resolution scanning tunneling microscope for a DLC film, as shown in Fig. 7. Despite the atomic smoothness, as measured by atomic force microscopy, the STM (scanning tunneling microscopy) results show that there are nanometer-sized regions of higher conductivity surrounded by other regions of lower conductivity as shown in Fig. 7. In this respect the model presented in Fig. 6 is able to explain the STM results. Increasing the concentration and size of the clusters results in improved localized electrical conductivity. This is an important result since conventional "large area" contacts used in current-voltage characteristics would mask the localized nature of the conductivity.

The implication of this "dielectric inhomogeneity" can also be extended to examine the field-induced electron emis- 


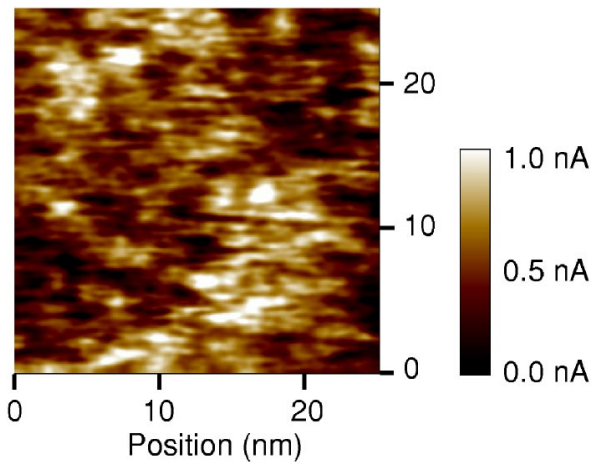

FIG. 7. (color online) High-resolution scanning tunneling microscope images of a DLC film deposited at $-265 \mathrm{~V}$ bias. The image shows localized regions of higher conductivity of several millimeters in diameter surrounded by less conductive regions.

sion from $a-\mathrm{C}: \mathrm{H}$ films. Application of an applied electric field can result in electron emission from the surface. In the case of PAC films it was previously reported ${ }^{32}$ that the threshold electric field for emission exhibited a dependence with the film thickness with a minimum threshold observed for a thickness of $65 \mathrm{~nm}$. This behavior was attributed to the effects of internal high electric field effects within the film and the absence of any significant screening of the applied electric field. Such an explanation is possible when one considers that the electric field from the anode will terminate on the "more conductive" parts of the films and if these are associated with the $s p^{2}$ clusters the low density $\left(\sim 10^{17} \mathrm{~cm}^{-3}\right)$ will be insufficient to screen the film/substrate interface from the applied field. In such a situation the controlling step in the electron emission process is at the film/ substrate interface. A different situation will result for films grown at higher biases when the defect density increases. For two films deposited at -90 and $-265 \mathrm{~V}$ self-bias, it was observed $^{33}$ that there is little dependence on the film thickness and the lowest threshold field was reported for the film grown at the highest bias, consistent with the model presented in Fig. 6. Here, the film with the largest bias has the greatest intercluster interactions since the clusters are larger and the cluster-cluster separation will be lowest. This gives rise to an emission mechanism that is dominated by the front surface properties of the film. Furthermore, the local electric field in the neighborhood of the clusters is enhanced, resulting in lower than predicted threshold fields for emission

It has been reported in several studies that the onset of stable field emission, often in more resistive samples, occurs only after several voltage cycles. ${ }^{32,34,35}$ This conditioning behavior often results in lower threshold fields. In a previous study we showed that it is possible to condition a PAC film by current stressing - that is, the intentional passing of a current through the film. ${ }^{35}$ Mercer et al. performed a similar experiment using a scanning tunneling microscope tip to generate a highly spatially localized electric field and effectively current stress $t a-\mathrm{C}$ films. ${ }^{36}$ They observed that after ramping the tip-sample bias and current, nanostructures of about $100 \mathrm{~nm}$ in extent form. By using high-resolution spatially resolved electron energy loss spectroscopy (EELS) they showed that the predominant bonding configuration changes from predominately fourfold coordinated $\mathrm{C}$ to threefold coordinated. The net effect of the conditioning or the current stressing treatments may well be to generate conductive $s p^{2}$-rich areas or filaments through localized Joule heating, reminiscent of the use of ion implantation to deposit energy locally.

\section{CONCLUSIONS}

In conclusion, different amorphous carbon thin films are characterized in terms of disorder and states near the Fermi level. PAC films have small clusters with a small distribution of cluster sizes and dominated by topological disorder. This is confirmed by the reduction of the Raman width at the higher excitation energies and the low spin density. PAC films possess low stress with long spin-lattice relaxation times. As a result in $a-\mathrm{C}: \mathrm{H}$ films, the Urbach energy is not a good measure of disorder, and topological disorder dominates. Moving from PAC to DLC films, by growing at higher negative self-biases the reduction in the Tauc gap and blue shift of the $G$ band implies that a higher concentration of larger clusters is present. Furthermore the increase in spin density $\left(10^{20} \mathrm{~cm}^{-3}\right)$ coupled with the increase in the $G$ band width implies more distorted clusters are present. The spinlattice relaxation time also shortens, implying that the paramagnetic electron states couple more strongly with the lattice. At the higher spin density the ESR linewidth is no longer determined by the dipole interaction, with intracluster exchange interactions determining the linewidth. The exchange interaction is also present at high microwave frequencies. In this regime both topological and structural disorder is present and can effect the stress in the film. The disorder is dominated by structural disorder with little topological disorder reduces thereby reducing linewidth of $G$ band.

\section{ACKNOWLEDGMENTS}

The authors would like to thank the EPSRC for funding via an Advanced Research Fellowship (J.D.C.) and via a Portfolio Partnership grant and the Carbon Based Electronics program.
*Electronic mail: David.Carey@surrey.ac.uk

${ }^{1}$ S. R. P. Silva, J. D. Carey, R. U. A. Khan, E. G. Gerstner, and J. V. Anguita, in Handbook of Thin Film Materials, edited by H. S. Nalwa, (Academic Press, New York, 2002), Vol. 4, Chap. 9, pp.
403-506.

${ }^{2}$ J. Robertson, Mater. Sci. Eng., R. 37, 129 (2002).

${ }^{3}$ S. R. P. Silva and J. D. Carey, Diamond Relat. Mater. 12, 151 (2003). 
${ }^{4}$ D. Dasgupta, F. Demichelis, C. F. Pirri, and A. Tagliaferro, Phys. Rev. B 43, 2131 (1991).

${ }^{5}$ G. Fanchini, S. C. Ray, and A Tagliaferro, Diamond Relat. Mater. 12, 891 (2003).

${ }^{6}$ A. C. Ferrari and J. Robertson, Phys. Rev. B 61, 14095 (2000).

${ }^{7}$ J. Ristein, J. Schäfer, and L. Ley, Diamond Relat. Mater. 4, 509 (1995).

${ }^{8}$ A. Zeinert, H-J von Bardeleben, and R. Bouzerar, Diamond Relat. Mater. 9, 728 (2000).

${ }^{9}$ F. Giorgis, F. Giuliani, C. F. Pirri, and A. Tagliaferro, Appl. Phys. Lett. 72, 2520 (1998).

${ }^{10}$ M. A. Tamor and W. C. Vassell, J. Appl. Phys. 76, 3823 (1994).

${ }^{11}$ R. C. Barklie, M. Collins, and S. R. P. Silva, Phys. Rev. B 61, 3546 (2000).

${ }^{12}$ M. Collins, R. C. Barklie, J. V. Anguita, J. D. Carey, and S. R. P. Silva, Diamond Relat. Mater. 9, 781 (2000).

${ }^{13}$ P. W. Anderson, Phys. Rev. 109, 1492 (1958).

${ }^{14}$ C. W. Chen and J. Robertson, J. Non-Cryst. Solids 227, 602 (1998).

${ }^{15}$ Rusli, J. Robertson, and G. A. J. Amaratunga, J. Appl. Phys. 80, 2998 (1996).

${ }^{16}$ S. R. P. Silva, J. Robertson, Rusli, G. A. J. Amaratunga, and J. Schwan, Philos. Mag. B 74, 369 (1996).

${ }^{17}$ G. Fanchini and A. Taliaferro, Appl. Phys. Lett. 85, 730 (2004).

${ }^{18}$ C. Jäger, J. Gottwald, H. W. Spiess, and R. J. Newport, Phys. Rev. B 50, 846 (1994).

${ }^{19}$ A. Abragam, in Principles of Nuclear Magnetism (Clarendon, Oxford, 1996), p. 126.

${ }^{20}$ M. A. Tamor, W. C. Vassell, and K. R. Carduner, Appl. Phys. Lett. 58, 592 (1991).

${ }^{21}$ A. Ilie, A. C. Ferrari, T. Yagi, and J. Robertson, Appl. Phys. Lett. 76, 2627 (2000).
${ }^{22}$ H. J. von Bardeleben, J. L. Cantin, A. Zeinert, B. Racine, K. Zellama, and P. N Hai, Appl. Phys. Lett. 78, 2843 (2001).

${ }^{23}$ G. Wagoner, Phys. Rev. 118, 647 (1960).

${ }^{24}$ B. Druz, I. Zaritskiy, Yu. Evtuikhov, A. Konchits, M. Ya Valakh, S. P. Kolesnik, B. D. Shanina, and V. Visotskij, Mater. Res. Soc. Symp. Proc. 593, 249 (2000).

${ }^{25}$ B. J. Jones, R. C. Barklie, G. Smith, H. El Mkami, J. D. Carey, and S. R. P. Silva, Diamond Relat. Mater. 12, 116 (2003).

${ }^{26}$ A. A. Konchits, M. Ya. Valakh, B. D. Shanina, S. P. Kolesnik, I. B. Yanchuk, J. D. Carey, and S. R. P. Silva, J. Appl. Phys. 93, 5905 (2003).

${ }^{27}$ M. P. Siegal, D. R. Tallant, P. N. Provencio, D. L. Overmyer, R. L. Simposn, and L. J. Martinez-Miranda, Appl. Phys. Lett. 76, 3052 (2000).

${ }^{28}$ P. C. Kelires, Phys. Rev. B 62, 15686 (2000).

${ }^{29}$ X. Chen, J. P. Sullivan, T. A. Friedmann, J. Murray Gibson, Appl. Phys. Lett. 84, 2823 (2004)

${ }^{30}$ G. A. J. Amaratunga and S. R. P. Silva, Appl. Phys. Lett. 68, 2529 (1996).

${ }^{31}$ R. U. A. Khan, J. D. Carey, S. R. P. Silva, B. J. Jones, and R. C. Barklie, Phys. Rev. B 63, 121201 (2001).

${ }^{32}$ R. D. Forrest, A. P. Burden, S. R. P. Silva, L. K. Cheah, and X. Shi, Appl. Phys. Lett. 73, 3784 (1998).

${ }^{33}$ J. D. Carey, R. D. Forrest, and S. R. P. Silva, Appl. Phys. Lett. 78, 2339 (2001).

${ }^{34}$ A. A. Talin, T. E. Felter, T. A. Friedmann, J. P. Sullivan, and M. P. Siegal, J. Vac. Sci. Technol. A 14, 1719 (1996).

${ }^{35}$ J. D. Carey and S. R. P. Silva, Appl. Phys. Lett. 78, 347 (2001).

${ }^{36}$ T. W. Mercer, N. J. DiNardo, J. B. Rothman, M. P. Siegal, T. A. Friedmann, and L. J. Martinez Miranda, Appl. Phys. Lett. 72, 2244 (1998). 